\title{
An Algebraic Analogue of a Formula of Knuth
}

\author{
Lionel Levine
}

Department of Mathematics, Massachusetts Institute of Technology, Cambridge, MA 02139

\begin{abstract}
We generalize a theorem of Knuth relating the oriented spanning trees of a directed graph $G$ and its directed line graph $\mathcal{L} G$. The sandpile group is an abelian group associated to a directed graph, whose order is the number of oriented spanning trees rooted at a fixed vertex. In the case when $G$ is regular of degree $k$, we show that the sandpile group of $G$ is isomorphic to the quotient of the sandpile group of $\mathcal{L} G$ by its $k$-torsion subgroup. As a corollary we compute the sandpile groups of two families of graphs widely studied in computer science, the de Bruijn graphs and Kautz graphs.

Résumé. Nous généralisons un théorème de Knuth qui relie les arbres couvrants dirigés d'un graphe orienté $G$ au graphe adjoint orienté $\mathcal{L} G$. On peut associer à tout graphe orienté un groupe abélien appelé groupe du tas de sable, et dont l'ordre est le nombre d'arbres couvrants dirigés enracinés en un sommet fixé. Lorsque $G$ est régulier de degré $k$, nous montrons que le groupe du tas de sable de $G$ est isomorphe au quotient du groupe du tas de sable de $\mathcal{L} G$ par son sous-groupe de $k$-torsion. Comme corollaire, nous déterminons les groupes de tas de sable de deux familles de graphes étudiées en informatique: les graphes de de Bruijn et les graphes de Kautz.
\end{abstract}

Keywords: critical group, de Bruijn graph, iterated line digraph, Kautz graph, matrix-tree theorem, oriented spanning tree, weighted Laplacian

\section{Introduction}

In this extended abstract we discuss some new generalizations of an enumerative formula of Knuth [10]. Proofs omitted here due to space constraints can be found in [11].

Let $G=(V, E)$ be a finite directed graph, which may have loops and multiple edges. Each edge $e \in E$ is directed from its source vertex $s(e)$ to its target vertex $t(e)$. The directed line graph $\mathcal{L} G=\left(E, E_{2}\right)$ has as vertices the edges of $G$, and as edges the set

$$
E_{2}=\left\{\left(e_{1}, e_{2}\right) \in E \times E \mid s\left(e_{2}\right)=t\left(e_{1}\right)\right\} .
$$

For example, if $G$ has just one vertex and $n$ loops, then $\mathcal{L} G$ is the complete directed graph on $n$ vertices. If $G$ has two vertices and no loops, then $\mathcal{L} G$ is a bidirected complete bipartite graph.

An oriented spanning tree of $G$ is a subgraph containing all of the vertices of $G$, having no directed cycles, in which one vertex, the root, has outdegree 0 , and every other vertex has outdegree 1 . The number $\kappa(G)$ of oriented spanning trees of $G$ is sometimes called the complexity of $G$.

Our first result relates the numbers $\kappa(\mathcal{L} G)$ and $\kappa(G)$. Let $\left\{x_{e}\right\}_{e \in E}$ and $\left\{x_{v}\right\}_{v \in V}$ be indeterminates, and consider the polynomials

1365-8050 @ 2010 Discrete Mathematics and Theoretical Computer Science (DMTCS), Nancy, France 


$$
\begin{gathered}
\kappa^{e d g e}(G, \mathbf{x})=\sum_{T} \prod_{e \in T} x_{e} \\
\kappa^{\text {vertex }}(G, \mathbf{x})=\sum_{T} \prod_{e \in T} x_{t(e)} .
\end{gathered}
$$

The sums are over all oriented spanning trees $T$ of $G$.

Write

$$
\begin{gathered}
\operatorname{indeg}(v)=\#\{e \in E \mid t(e)=v\} \\
\operatorname{outdeg}(v)=\#\{e \in E \mid s(e)=v\}
\end{gathered}
$$

for the indegree and outdegree of vertex $v$ in $G$. We say that $v$ is a source if $\operatorname{indeg}(v)=0$.

Theorem 1.1 Let $G=(V, E)$ be a finite directed graph with no sources. Then

$$
\kappa^{\text {vertex }}(\mathcal{L} G, \mathbf{x})=\kappa^{\text {edge }}(G, \mathbf{x}) \prod_{v \in V}\left(\sum_{s(e)=v} x_{e}\right)^{\operatorname{indeg}(v)-1}
$$

Note that since the vertex set of $\mathcal{L} G$ coincides with the edge set of $G$, both sides of (1) are polynomials in the same set of variables $\left\{x_{e}\right\}_{e \in E}$. Setting all $x_{e}=1$ yields the product formula

$$
\kappa(\mathcal{L} G)=\kappa(G) \prod_{v \in V} \operatorname{outdeg}(v)^{\operatorname{indeg}(v)-1}
$$

due in a slightly different form to Knuth [10]. Special cases of 22] include Cayley's formula $n^{n-1}$ for the number of rooted spanning trees of the complete graph $K_{n}$, as well as the formula $(m+n) m^{n-1} n^{m-1}$ for the number of rooted spanning trees of the complete bipartite graph $K_{m, n}$. These are respectively the cases that $G$ has just one vertex with $n$ loops, or $G$ has just two vertices $a$ and $b$ with $m$ edges directed from $a$ to $b$ and $n$ edges directed from $b$ to $a$.

Suppose now that $G$ is strongly connected, that is, for any $v, w \in V$ there are directed paths in $G$ from $v$ to $w$ and from $w$ to $v$. Then associated to any vertex $v_{*}$ of $G$ is an abelian group $K\left(G, v_{*}\right)$, the sandpile group, whose order is the number of oriented spanning trees of $G$ rooted at $v_{*}$. Its definition and basic properties are reviewed in section 3 . Other common names for this group are the critical group, Picard group, Jacobian, and group of components. In the case when $G$ is Eulerian (that is, $\operatorname{indeg}(v)=$ $\operatorname{outdeg}(v)$ for all vertices $v$ ) the groups $K\left(G, v_{*}\right)$ and $K\left(G, v_{*}^{\prime}\right)$ are isomorphic for any $v_{*}, v_{*}^{\prime} \in V$, and we often denote the sandpile group just by $K(G)$.

When $G$ is Eulerian, we show that there is a natural map from the sandpile group of $\mathcal{L} G$ to the sandpile group of $G$, descending from the $\mathbb{Z}$-linear map

$$
\phi: \mathbb{Z}^{E} \rightarrow \mathbb{Z}^{V}
$$

which sends $e \mapsto t(e)$.

Let $k$ be a positive integer. We say that $G$ is balanced $k$-regular if $\operatorname{indeg}(v)=\operatorname{outdeg}(v)=k$ for every vertex $v$. 
Theorem 1.2 Let $G=(V, E)$ be a strongly connected Eulerian directed graph, fix $e_{*} \in E$ and let $v_{*}=t\left(e_{*}\right)$. The map $\phi$ descends to a surjective group homomorphism

$$
\bar{\phi}: K\left(\mathcal{L} G, e_{*}\right) \rightarrow K\left(G, v_{*}\right) .
$$

Moreover, if $G$ is balanced $k$-regular, then $\operatorname{ker}(\bar{\phi})$ is the $k$-torsion subgroup of $K\left(\mathcal{L} G, e_{*}\right)$.

This result extends to directed graphs some of the recent work of Berget, Manion, Maxwell, Potechin and Reiner [1] on undirected line graphs. If $G=(V, E)$ is an undirected graph, the (undirected) line graph line $(G)$ of $G$ has vertex set $E$ and edge set

$$
\left\{\left\{e, e^{\prime}\right\} \mid e, e^{\prime} \in E, e \cap e^{\prime} \neq \emptyset\right\} .
$$

The results of [1] relate the sandpile groups of $G$ and line $(G)$. The undirected case is considerably more subtle, because although there is still a natural map $K($ line $G) \rightarrow K(G)$ when $G$ is regular, this map may fail to be surjective.

A particularly interesting family of directed line graphs are the de Bruijn graphs $D B_{n}$, defined recursively by

$$
D B_{n}=\mathcal{L}\left(D B_{n-1}\right), \quad n \geq 1,
$$

where $D B_{0}$ is the graph with just one vertex and two loops. The $2^{n}$ vertices of $D B_{n}$ can be identified with binary words $b_{1} \ldots b_{n}$ of length $n$; two such sequences $b$ and $b^{\prime}$ are joined by a directed edge $\left(b, b^{\prime}\right)$ if and only if $b_{i}^{\prime}=b_{i+1}$ for all $i=1, \ldots, n-1$.

Using Theorem 1.2, we obtain the full structure of the sandpile groups of the de Bruijn graphs.

\section{Theorem 1.3}

$$
K\left(D B_{n}\right)=\bigoplus_{j=1}^{n-1}\left(\mathbb{Z} / 2^{j} \mathbb{Z}\right)^{2^{n-1-j}} .
$$

Closely related to the de Bruijn graphs are the Kautz graphs, defined by

$$
\text { Kautz }_{1}=(\{1,2,3\},\{(1,2),(1,3),(2,1),(2,3),(3,1),(3,2)\})
$$

and

$$
\text { Kautz }_{n}=\mathcal{L}\left(\text { Kautz }_{n-1}\right), \quad n \geq 2 .
$$

The Kautz graphs are useful in network design because they have close to the maximum possible number of vertices given their diameter and degree [7] and because they contain many short vertex-disjoint paths between any pair of vertices [5]. The following result gives the sandpile group of Kautz ${ }_{n}$.

\section{Theorem 1.4}

$$
K\left(\operatorname{Kautz}_{n}\right)=(\mathbb{Z} / 3 \mathbb{Z}) \oplus\left(\mathbb{Z} / 2^{n-1} \mathbb{Z}\right)^{2} \oplus \bigoplus_{j=1}^{n-2}\left(\mathbb{Z} / 2^{j} \mathbb{Z}\right)^{3 \cdot 2^{n-2-j}} .
$$

Bidkhori and Kishore [2] have recently generalized Theorems 1.3 and 1.4 to $m$-regular de Bruijn and Kautz graphs.

The remainder of the paper is organized as follows. In section 2, we discuss some interesting variants and special cases of Theorem 1.1 . Section 3 begins by defining the sandpile group, and moves on from 
there to the proof of Theorem 1.2 In section 4 we enumerate spanning trees of iterated line digraphs. Huaxiao, Fuji and Qiongxiang [9] prove that for a balanced $k$-regular directed graph $G$ on $N$ vertices,

$$
\kappa\left(\mathcal{L}^{n} G\right)=\kappa(G) k^{\left(k^{n}-1\right) N} .
$$

Theorem 4.1 generalizes this formula to an arbitrary directed graph $G$ having no sources. Section 4 also contains the proofs of Theorems 1.3 and 1.4

\section{Spanning Trees}

In this section we discuss a few variants and special cases of Theorem 1.1. We omit the proof due to space constraints. See [11] for a proof using the matrix-tree theorem. Very recently, Bidkhori and Kishore [2] have found a bijective proof, and used it to resolve a question of Stanley about de Bruijn sequences.

Theorem 1.1 enumerates all oriented spanning trees of $\mathcal{L} G$, while in many applications one wants to enumerate spanning trees with a fixed root. Given a vertex $v_{*} \in V$, let

$$
\kappa^{e d g e}\left(G, v_{*}, \mathbf{x}\right)=\sum_{\operatorname{root}(T)=v_{*}} \prod_{e \in T} x_{e}
$$

and

$$
\kappa^{\text {vertex }}\left(G, v_{*}, \mathbf{x}\right)=\sum_{\operatorname{root}(T)=v_{*}} \prod_{e \in T} x_{t(e)} .
$$

The following variant of Theorem 1.1 enumerates spanning trees of $\mathcal{L} G$ with a fixed root $e_{*}$ in terms of spanning trees of $G$ with root $w_{*}=s\left(e_{*}\right)$.

Theorem 2.1 Let $G=(V, E)$ be a finite directed graph, and let $e_{*}=\left(w_{*}, v_{*}\right)$ be an edge of $G$. If $\operatorname{indeg}(v) \geq 1$ for all vertices $v \in V$, and $\operatorname{indeg}\left(v_{*}\right) \geq 2$, then

$$
\begin{aligned}
& \kappa^{\text {vertex }}\left(\mathcal{L} G, e_{*}, \mathbf{x}\right)=\kappa^{e d g e}\left(G, w_{*}, \mathbf{x}\right) x_{e_{*}}\left(\sum_{s(e)=v_{*}} x_{e}\right)^{\operatorname{indeg}\left(v_{*}\right)-2} \times \\
& \times \prod_{v \neq v_{*}}\left(\sum_{s(e)=v} x_{e}\right)^{\operatorname{indeg}(v)-1} .
\end{aligned}
$$

Setting all $x_{e}=1$ in Theorem 2.1 yields the enumeration

$$
\kappa\left(\mathcal{L} G, e_{*}\right)=\frac{\kappa\left(G, w_{*}\right)}{\operatorname{outdeg}\left(v_{*}\right)} \pi(G)
$$

where $\kappa\left(G, w_{*}\right)$ is the number of oriented spanning trees of $G$ rooted at $w_{*}$, and

$$
\pi(G)=\prod_{v \in V} \operatorname{outdeg}(v)^{\operatorname{indeg}(v)-1} .
$$


It is interesting to compare this formula to the theorem of Knuth [10], which in our notation reads

$$
\kappa\left(\mathcal{L} G, e_{*}\right)=\left(\kappa\left(G, v_{*}\right)-\frac{1}{\operatorname{outdeg}\left(v_{*}\right)} \sum_{\substack{t(e)=v_{*} \\ e \neq e_{*}}} \kappa(G, s(e))\right) \pi(G) .
$$

To see directly why the right sides of (3) and (4) are equal, we define a unicycle to be a spanning subgraph of $G$ which contains a unique directed cycle, and in which every vertex has outdegree 1 . If vertex $v_{*}$ is on the unique cycle of a unicycle $U$, we say that $U$ goes through $v_{*}$.

\section{Lemma 2.2}

$$
\kappa^{e d g e}\left(G, v_{*}, \mathbf{x}\right) \sum_{s(e)=v_{*}} x_{e}=\sum_{t(e)=v_{*}} \kappa^{e d g e}(G, s(e), \mathbf{x}) x_{e} .
$$

Proof: Removing $e$ gives a bijection from unicycles containing a fixed edge $e$ to spanning trees rooted at $s(e)$. If $U$ is a unicycle through $v_{*}$, then the cycle of $U$ contains a unique edge $e$ with $s(e)=v_{*}$ and a unique edge $e^{\prime}$ with $t\left(e^{\prime}\right)=v_{*}$, so both sides are equal to

$$
\sum_{U} \prod_{e \in U} x_{e}
$$

where the sum is over all unicycles $U$ through $v_{*}$.

Setting all $x_{e}=1$ in Lemma 2.2 yields

$$
\operatorname{outdeg}\left(v_{*}\right) \kappa\left(G, v_{*}\right)=\sum_{t(e)=v_{*}} \kappa(G, s(e)) .
$$

Hence the factor appearing in front of $\pi(G)$ in Knuth's formula $\sqrt{4}$ is equal to $\kappa\left(G, w_{*}\right) / \operatorname{outdeg}\left(v_{*}\right)$.

We conclude this section by discussing some interesting examples and special cases of Theorem 1.1 .

- Deletion and contraction. Fix $e \in E$ and set $x_{f}=1$ for all $f \neq e$. The coefficient of $x_{e}^{\ell}$ in $\kappa^{\text {vertex }}(\mathcal{L} G, \mathbf{x})$ then counts the number of oriented spanning trees $T$ of $\mathcal{L} G$ with $\operatorname{indeg}_{T}(e)=\ell$. If $v=s(e)$ has indegree $k$ and outdegree $m$, then this coefficient is given by

$$
\begin{aligned}
\prod_{w \neq v} \operatorname{outdeg}(w)^{\operatorname{indeg}(w)-1} & \left(\begin{array}{c}
k-1 \\
\ell
\end{array}\right) \kappa(G \backslash e)(m-1)^{k-1-\ell}+ \\
& \left.+\left(\begin{array}{c}
k-1 \\
\ell-1
\end{array}\right) \kappa(G / e)(m-1)^{k-\ell}\right) .
\end{aligned}
$$

Here $G \backslash e$ and $G / e$ are respectively the graphs resulting from deleting and contracting the edge $e$. (There is more than one sensible way to define contraction for directed graphs. By $G / e$ we mean the graph obtained from $G$ by first deleting all edges $f$ with $s(f)=s(e)$, and then identifying the vertices $s(e)$ and $t(e)$.) 
- Complete graph. Taking $G$ to be the graph with one vertex and $n$ loops, so that $\mathcal{L} G$ is the complete directed graph $\vec{K}_{n}$ on $n$ vertices, we obtain the classical formula

$$
\kappa^{\text {vertex }}\left(\vec{K}_{n}\right)=\left(x_{1}+\ldots+x_{n}\right)^{n-1} .
$$

For a generalization to forests, see [15, Theorem 5.3.4]. Note that oriented spanning trees of $\vec{K}_{n}$ are in bijection with rooted spanning trees of the complete undirected graph $K_{n}$, by forgetting orientation.

- Complete bipartite graph. Taking $G$ to have two vertices, $a$ and $b$, with $m$ edges directed from $a$ to $b$ and $n$ edges directed from $b$ to $a$, we obtain

$$
\begin{aligned}
& \kappa^{\text {vertex }}\left(\vec{K}_{m, n}\right)=\left(x_{1}+\ldots+x_{m}\right)^{n-1}\left(y_{1}+\ldots+y_{n}\right)^{m-1} . \\
& \cdot\left(x_{1}+\ldots+x_{m}+y_{1}+\ldots+y_{n}\right)
\end{aligned}
$$

where $\vec{K}_{m, n}=\mathcal{L} G$ is the bidirected complete bipartite graph on $m+n$ vertices. The variables $x_{1}, \ldots, x_{m}$ correspond to vertices in the first part, and $y_{1}, \ldots, y_{n}$ correspond to vertices in the second part. As with the complete graph, oriented spanning trees of $\vec{K}_{m, n}$ are in bijection with rooted spanning trees of the undirected complete bipartite graph $K_{m, n}$ by forgetting orientation.

- De Bruijn graphs. The spanning tree enumerators for the first few de Bruijn graphs are

$$
\begin{gathered}
\kappa^{\text {vertex }}\left(D B_{1}\right)=x_{0}+x_{1} ; \\
\kappa^{\text {vertex }}\left(D B_{2}\right)=\left(x_{00}+x_{01}\right)\left(x_{10}+x_{11}\right)\left(x_{01}+x_{10}\right) ; \\
\kappa^{\text {vertex }}\left(D B_{3}\right)=\left(x_{000}+x_{001}\right)\left(x_{010}+x_{011}\right)\left(x_{100}+x_{101}\right)\left(x_{110}+x_{111}\right) \times \\
\times\left(x_{011} x_{110} x_{100}+x_{010} x_{110} x_{100}+x_{110} x_{101} x_{001}+x_{110} x_{100} x_{001}+\right. \\
\left.+x_{100} x_{001} x_{011}+x_{101} x_{001} x_{011}+x_{001} x_{010} x_{110}+x_{001} x_{011} x_{110}\right) .
\end{gathered}
$$

\section{Sandpile Groups}

Let $G=(V, E)$ be a strongly connected finite directed graph, loops and multiple edges allowed. Consider the free abelian group $\mathbb{Z}^{V}$ generated by the vertices of $G$; we think of its elements as formal linear combinations of vertices with integer coefficients. For $v \in V$ let

$$
\Delta_{v}=\sum_{s(e)=v}(t(e)-v) \in \mathbb{Z}^{V}
$$

where the sum is over all edges $e \in E$ such that $s(e)=v$. Fixing a vertex $v_{*} \in V$, let $L_{V}$ be the subgroup of $\mathbb{Z}^{V}$ generated by $v_{*}$ and $\left\{\Delta_{v}\right\}_{v \neq v_{*}}$. The sandpile group $K\left(G, v_{*}\right)$ is defined as the quotient group

$$
K\left(G, v_{*}\right)=\mathbb{Z}^{V} / L_{V} .
$$


The $V \times V$ integer matrix whose column vectors are $\left\{\Delta_{v}\right\}_{v \in V}$ is called the Laplacian of $G$. Its principal minor omitting the row and column corresponding to $v_{*}$ counts the number $\kappa\left(G, v_{*}\right)$ of oriented spanning trees of $G$ rooted at $v_{*}$. (This is the matrix-tree theorem, [15, Theorem 5.6.4].) Since this minor is also the index of $L_{V}$ in $\mathbb{Z}^{V}$, we have

$$
\# K\left(G, v_{*}\right)=\kappa\left(G, v_{*}\right)
$$

Recall that $G$ is Eulerian if $\operatorname{indeg}(v)=\operatorname{outdeg}(v)$ for every vertex $v$. If $G$ is Eulerian, then the groups $K\left(G, v_{*}\right)$ and $K\left(G, v_{*}^{\prime}\right)$ are isomorphic for any vertices $v_{*}$ and $v_{*}^{\prime}$ [8, Lemma 4.12]. In this case we usually denote the sandpile group just by $K(G)$.

The sandpile group arose independently in several fields, including arithmetic geometry [12, 13], statistical physics [4] and algebraic combinatorics [3]. Often it is defined for an undirected graph $G$; to translate this definition into the present setting of directed graphs, replace each undirected edge by a pair of directed edges oriented in opposite directions. Sandpiles on directed graphs were first studied in [14]. For a survey of the basic properties of sandpile groups of directed graphs and their proofs, see [8].

The goal of this section is to relate the sandpile groups of an Eulerian graph $G$ and its directed line graph $\mathcal{L} G$. To that end, let $\mathbb{Z}^{E}$ be the free abelian group generated by the edges of $G$. For $e \in E$ let

$$
\Delta_{e}=\sum_{s(f)=t(e)}(f-e) \in \mathbb{Z}^{E}
$$

Fix an edge $e_{*} \in E$, and let $v_{*}=t\left(e_{*}\right)$. Let $L_{E} \subset \mathbb{Z}^{E}$ be the subgroup generated by $e_{*}$ and $\left\{\Delta_{e}\right\}_{e \neq e_{*}}$. Then the sandpile group associated to $\mathcal{L} G$ and $e_{*}$ is

$$
K\left(\mathcal{L} G, e_{*}\right)=\mathbb{Z}^{E} / L_{E}
$$

Note that $\mathcal{L} G$ may not be Eulerian even when $G$ is Eulerian.

Lemma 3.1 Let $\phi: \mathbb{Z}^{E} \rightarrow \mathbb{Z}^{V}$ be the $\mathbb{Z}$-linear map sending $e \mapsto t(e)$. If $G$ is Eulerian, then $\phi$ descends to a surjective group homomorphism

$$
\bar{\phi}: K\left(\mathcal{L} G, e_{*}\right) \rightarrow K\left(G, v_{*}\right)
$$

Proof: To show that $\phi$ descends, it suffices to show that $\phi\left(L_{E}\right) \subset L_{V}$. For any $e \in E$, we have

$$
\phi\left(\Delta_{e}\right)=\sum_{s(f)=t(e)}(t(f)-t(e))=\Delta_{t(e)}
$$

The right side lies in $L_{V}$ by definition if $t(e) \neq v_{*}$. Moreover, since $G$ is Eulerian,

$$
\sum_{v \in V} \Delta_{v}=\sum_{e \in E}(t(e)-s(e))=\sum_{v \in V}(\operatorname{indeg}(v)-\operatorname{outdeg}(v)) v=0
$$

so $\Delta_{v_{*}}=-\sum_{v \neq v_{*}} \Delta_{v}$ also lies in $L_{V}$. Finally, $\phi\left(e_{*}\right)=v_{*} \in L_{V}$, and hence $\phi\left(L_{E}\right) \subset L_{V}$.

Since $G$ is strongly connected, every vertex has at least one incoming edge, so $\phi$ is surjective, and hence $\bar{\phi}$ is surjective. 
Let $k$ be a positive integer. We say that $G$ is balanced $k$-regular if indeg $(v)=\operatorname{outdeg}(v)=k$ for every vertex $v$. Note that any balanced $k$-regular graph is Eulerian; and if $G$ is balanced $k$-regular, then its directed line graph $\mathcal{L} G$ is also balanced $k$-regular. In particular, this implies

$$
\sum_{e \in E} \Delta_{e}=0
$$

so that $\Delta_{e_{*}} \in L_{E}$.

Now consider the $\mathbb{Z}$-linear map

$$
\psi: \mathbb{Z}^{V} \rightarrow \mathbb{Z}^{E}
$$

sending $v \mapsto \sum_{s(e)=v} e$. For a group $\Gamma$, write $k \Gamma=\{k g \mid g \in \Gamma\}$. The following lemma is proved in [11].

Lemma 3.2 If $G$ is balanced $k$-regular, then $\psi$ descends to a group isomorphism

$$
\bar{\psi}: K(G) \stackrel{\simeq}{\rightarrow} k K(\mathcal{L} G) .
$$

Proof of Theorem 1.2 If $G$ is Eulerian, then $\phi$ descends to a surjective homomorphism of sandpile groups by Lemma 3.1. If $G$ is balanced $k$-regular, then $\bar{\psi}$ is injective by Lemma 3.2, so

$$
\operatorname{ker}(\bar{\phi})=\operatorname{ker}(\bar{\psi} \circ \bar{\phi}) \text {. }
$$

Moreover for any edge $e \in E$

$$
(\psi \circ \phi)(e)=\sum_{s(f)=t(e)} f=k e+\Delta_{e} .
$$

Hence $\bar{\psi} \circ \bar{\phi}$ is multiplication by $k$, and $\operatorname{ker}(\bar{\phi})$ is the $k$-torsion subgroup of $K(\mathcal{L} G)$.

\section{Iterated Line Graphs}

Let $G=(V, E)$ be a finite directed graph, loops and multiple edges allowed. The iterated line digraph $\mathcal{L}^{n} G=\left(E_{n}, E_{n+1}\right)$ has as vertices the set

$$
E_{n}=\left\{\left(e_{1}, \ldots, e_{n}\right) \in E^{n} \mid s\left(e_{i+1}\right)=t\left(e_{i}\right), i=1, \ldots, n-1\right\}
$$

of directed paths of $n$ edges in $G$. The edge set of $\mathcal{L}^{n} G$ is $E_{n+1}$, and the incidence is defined by

$$
\begin{aligned}
& s\left(e_{1}, \ldots, e_{n+1}\right)=\left(e_{1}, \ldots, e_{n}\right) ; \\
& t\left(e_{1}, \ldots, e_{n+1}\right)=\left(e_{2}, \ldots, e_{n+1}\right) .
\end{aligned}
$$

(We also set $E_{0}=V$, and $\mathcal{L}^{0} G=G$.) For example, the de Bruijn graph $D B_{n}$ is $\mathcal{L}^{n}\left(D B_{0}\right)$, where $D B_{0}$ is the graph with one vertex and two loops.

Our next result relates the number of spanning trees of $G$ and $\mathcal{L}^{n} G$. Given a vertex $v \in V$, let

$$
p(n, v)=\#\left\{\left(e_{1}, \ldots, e_{n}\right) \in E_{n} \mid t\left(e_{n}\right)=v\right\}
$$

be the number of directed paths of $n$ edges in $G$ ending at vertex $v$. 
Theorem 4.1 Let $G=(V, E)$ be a finite directed graph with no sources. Then

$$
\kappa\left(\mathcal{L}^{n} G\right)=\kappa(G) \prod_{v \in V} \operatorname{outdeg}(v)^{p(n, v)-1} .
$$

Proof: For any $j \geq 0$, by Theorem 1.1 applied to $\mathcal{L}^{j} G$ with all edge weights 1 ,

$$
\begin{aligned}
\frac{\kappa\left(\mathcal{L}^{j+1} G\right)}{\kappa\left(\mathcal{L}^{j} G\right)} & =\prod_{\left(e_{1}, \ldots, e_{j}\right) \in E_{j}} \operatorname{outdeg}\left(t\left(e_{j}\right)\right)^{\operatorname{indeg}\left(s\left(e_{1}\right)\right)-1} \\
& =\prod_{v \in V} \operatorname{outdeg}(v)^{p(j+1, v)-p(j, v)}
\end{aligned}
$$

Taking the product over $j=0, \ldots, n-1$ yields the result.

When $G$ is balanced $k$-regular, we have $p(n, v)=k^{n}$ for all vertices $v$, so we obtain as a special case of Theorem 4.1 the result of Huaxiao, Fuji and Qiongxiang [9, Theorem 1]

$$
\kappa\left(\mathcal{L}^{n} G\right)=\kappa(G) k^{\left(k^{n}-1\right) \# V} .
$$

In particular, taking $G=D B_{0}$ yields the classical formula

$$
\kappa\left(D B_{n}\right)=2^{2^{n}-1} .
$$

Since $D B_{n}$ is Eulerian, the number $\kappa\left(D B_{n}, v_{*}\right)$ of oriented spanning trees rooted at $v_{*}$ does not depend on $v_{*}$, so

$$
\kappa\left(D B_{n}, v_{*}\right)=2^{-n} \kappa\left(D B_{n}\right)=2^{2^{n}-n-1} .
$$

This familiar number counts de Bruijn sequences of order $n+1$ (Eulerian tours of $D B_{n}$ ) up to cyclic equivalence. De Bruijn sequences are in bijection with oriented spanning trees of $D B_{n}$ rooted at a fixed vertex $v_{*}$; for more on the connection between spanning trees and Eulerian tours, see [6] and [15, section 5.6].

Perhaps less familiar is the situation when $G$ is not regular. As an example, consider the graph

$$
G=(\{0,1\},\{(0,0),(0,1),(1,0)\}) .
$$

The vertices of its iterated line graph $\mathcal{L}^{n} G$ are binary words of length $n+1$ containing no two consecutive 1's. The number of such words is the Fibonacci number $F_{n+3}$, and the number of words ending in 0 is $F_{n+2}$. By Theorem 4.1, the number of oriented spanning trees of $\mathcal{L}^{n} G$ is

$$
\kappa\left(\mathcal{L}^{n} G\right)=2 \cdot 2^{p(n, 0)-1}=2^{F_{n+2}} .
$$

Next we turn to the proofs of Theorems 1.3 and 1.4 . If $a$ and $b$ are positive integers, we write $\mathbb{Z}_{b}^{a}$ for the group $(\mathbb{Z} / b \mathbb{Z}) \oplus \ldots \oplus(\mathbb{Z} / b \mathbb{Z})$ with $a$ summands.

Proof of Theorem 1.3; Induct on $n$. From (5) we have

$$
\# K\left(D B_{n}\right)=2^{2^{n}-n-1}
$$


hence

$$
K\left(D B_{n}\right)=\mathbb{Z}_{2}^{a_{1}} \oplus \mathbb{Z}_{4}^{a_{2}} \oplus \mathbb{Z}_{8}^{a_{3}} \oplus \ldots \oplus \mathbb{Z}_{2^{m}}^{a_{m}}
$$

for some nonnegative integers $m$ and $a_{1}, \ldots, a_{m}$ satisfying

$$
\sum_{j=1}^{m} j a_{j}=2^{n}-n-1
$$

By Lemma 3.2 and the inductive hypothesis,

$$
\begin{aligned}
\mathbb{Z}_{2}^{a_{2}} \oplus \mathbb{Z}_{4}^{a_{3}} \oplus \ldots \oplus \mathbb{Z}_{2^{m-1}}^{a_{m}} & \simeq 2 K\left(D B_{n}\right) \\
& \simeq K\left(D B_{n-1}\right) \\
& \simeq \mathbb{Z}_{2}^{2^{n-3}} \oplus \mathbb{Z}_{4}^{2^{n-4}} \oplus \ldots \oplus \mathbb{Z}_{2^{n-2}}
\end{aligned}
$$

hence $m=n-1$ and

$$
a_{2}=2^{n-3}, a_{3}=2^{n-4}, \ldots, a_{n-1}=1 .
$$

Solving (6) for $a_{1}$ now yields $a_{1}=2^{n-2}$.

For $p$ prime, by carrying out the same argument on a general balanced $p$-regular directed graph $G$ on $N$ vertices, we find that

$$
K\left(\mathcal{L}^{n} G\right) \simeq \tilde{K} \oplus \bigoplus_{j=1}^{n-1}\left(\mathbb{Z}_{p^{j}}\right)^{p^{n-1-j}(p-1)^{2} N} \oplus\left(\mathbb{Z}_{p^{n}}\right)^{(p-1) N-r-1} \oplus \bigoplus_{j=1}^{m}\left(\mathbb{Z}_{p^{n+j}}\right)^{a_{j}}
$$

where

$$
\begin{gathered}
\operatorname{Sylow}_{p}(K(G))=\left(\mathbb{Z}_{p}\right)^{a_{1}} \oplus \ldots \oplus\left(\mathbb{Z}_{p^{m}}\right)^{a_{m}} ; \\
\tilde{K}=K(G) / \operatorname{Sylow}_{p}(K(G)) ; \\
r=a_{1}+\ldots+a_{m} .
\end{gathered}
$$

In particular, taking $G=$ Kautz $_{1}$ with $p=2$, we have $K(G)=\tilde{K}=\mathbb{Z}_{3}$, and we arrive at Theorem 1.4

\section{Concluding Remark}

Theorem 1.2 describes a map from the sandpile group $K\left(\mathcal{L} G, e_{*}\right)$ to the group $K\left(G, v_{*}\right)$ when $G$ is an Eulerian directed graph and $e_{*}=\left(w_{*}, v_{*}\right)$ is an edge of $G$. There is also a suggestive numerical relationship between the orders of the sandpile groups $K\left(\mathcal{L} G, e_{*}\right)$ and $K\left(G, w_{*}\right)$, which holds even when $G$ is not Eulerian: by equation (3) we have

$$
\kappa\left(G, w_{*}\right) \mid \kappa\left(\mathcal{L} G, e_{*}\right)
$$

whenever $G$ satisfies the hypothesis of Theorem 2.1. This observation leads us to ask whether $K\left(G, w_{*}\right)$ can be expressed as a subgroup or quotient group of $K\left(\mathcal{L} G, e_{*}\right)$. 


\section{References}

[1] A. Berget, A. Manion, M. Maxwell, A. Potechin and V. Reiner, The critical group of a line graph, Ann. Combin., to appear.http://arxiv.org/abs/0904.1246.

[2] H. Bidkhori and S. Kishore, Counting the spanning trees of a directed line graph. http://arxiv. org/abs/0910.3442

[3] N. L. Biggs, Chip-firing and the critical group of a graph, J. Algebraic Combin. 9, no. 1 (1999), $25-45$.

[4] D. Dhar, Self-organized critical state of sandpile automaton models, Phys. Rev. Lett. 64 (1990), 1613-1616.

[5] D.-Z. Du, Y.-D. Lyuu, and F. D. Hsu, Line digraph iterations and connectivity analysis of de Bruijn and Kautz graphs, IEEE Trans. Comput. 42, no. 5 (1993), 612-616.

[6] T. van Aardenne-Ehrenfest and N. G. de Bruijn, Circuits and trees in oriented linear graphs, Simon Stevin 28 (1951), 203-217.

[7] M. A. Fiol, J. L. A. Yebra and I. A. De Miquel, Line digraph iterations and the $(d, k)$-digraph problem, IEEE Trans. Comput. 33, no. 5 (1984), 400-403.

[8] A. E. Holroyd, L. Levine, K. Mészáros, Y. Peres, J. Propp and D. B. Wilson, Chip-firing and rotorrouting on directed graphs, In and out of equilibrium 2, 331-364, Progr. Probab. 60, Birkhäuser, 2008. http://arxiv.org/abs/0801.3306

[9] Z. Huaxiao, Z. Fuji and H. Qiongxiang, On the number of spanning trees and Eulerian tours in iterated line digraphs, Discrete Appl. Math. 73, no. 1 (1997), 59-67.

[10] D. E. Knuth, Oriented subtrees of an arc digraph, J. Comb. Theory 3 (1967), 309-314.

[11] L. Levine, Sandpile groups and spanning trees of directed line graphs, J. Comb. Theory A, to appear. http://arxiv.org/abs/0906.2809

[12] D. J. Lorenzini, Arithmetical graphs, Math. Ann. 285, no. 3 (1989), 481-501.

[13] D. J. Lorenzini, A finite group attached to the Laplacian of a graph, Discrete Math. 91, no. 3 (1991), 277-282.

[14] E. R. Speer, Asymmetric abelian sandpile models. J. Statist. Phys. 71 (1993), 61-74.

[15] R. P. Stanley, Enumerative Combinatorics, vol. 2, Cambridge University Press, 1999. 
